\title{
Three Dimensional Alternating-Phase Focusing for Dielectric-Laser Electron Accelerators
}

\author{
Uwe Niedermayer $\odot,^{1^{*}}$ Thilo Egenolf $\odot,{ }^{1}$ and Oliver Boine-Frankenheim ${ }^{1,2}$ \\ ${ }^{1}$ Technische Universität Darmstadt, Schlossgartenstrasse 8, D-64289 Darmstadt, Germany \\ ${ }^{2}$ GSI Helmholtzzentrum für Schwerionenforschung GmbH, Planckstrasse 1, D-64291 Darmstadt, Germany
}

(Received 11 April 2020; revised 21 August 2020; accepted 9 September 2020; published 13 October 2020)

\begin{abstract}
The concept of dielectric-laser acceleration (DLA) provides the highest gradients among breakdownlimited (nonplasma) particle accelerators and thus the potential of miniaturization. The implementation of a fully scalable electron accelerator on a microchip by two-dimensional alternating phase focusing (APF), which relies on homogeneous laser fields and external magnetic focusing in the third direction, was recently proposed. In this Letter, we generalize the APF for DLA scheme to 3D, such that stable beam transport and acceleration is attained without any external equipment, while the structures can still be fabricated by entirely two-dimensional lithographic techniques. In the new scheme, we obtain significantly higher accelerating gradients at given incident laser field by additionally exploiting the new horizontal edge. This enables ultralow injection energies of about $2.5 \mathrm{keV}(\beta=0.1)$ and bulky high voltage equipment as used in previous DLA experiments can be omitted. DLAs have applications in ultrafast time-resolved electron microscopy and diffraction. Our findings are crucial for the miniaturization of the entire setup and pave the way towards integration of DLAs in optical fiber driven endoscopes, e.g., for medical purposes.
\end{abstract}

DOI: 10.1103/PhysRevLett.125.164801

Dielectric laser acceleration (DLA) was already proposed in 1962 [1,2], however, first experiments came 50 years later $[3,4]$ by means of femtosecond laser pulses and lithographic nanofabrication. Recent advances in ultrashort laser pulses have enabled demonstrations of damagethreshold and self-phase modulation limited record peak gradients approaching the $\mathrm{GeV} / \mathrm{m}$ milestone for relativistic electrons [5,6]. At subrelativistic energies, driven by a tabletop electrostatic preaccelerator, peak gradients of 133 [7] and $370 \mathrm{MeV} / \mathrm{m}$ [8] were achieved in silicon pillar structures. In order to create a functioning accelerator out of these impressive gradients, the interaction length needs to be increased while maintaining a stable $6 \mathrm{D}$ phase space confinement. Such a miniaturized accelerator has applications in attosecond science $[9,10]$, ultrafast electron microscopy and diffraction (UEM/UED) [11-15], lithography [16], and it would be possible to provide relativistic electrons within (e.g., medical) endoscopes [17].

First approaches to beam dynamics in grating linacs were already made in the 1980s [18-20]. In 2012, Naranjo et al. [21] showed that the nonsynchronous spatial (sub)harmonics can provide a ponderomotive focusing force, which was later turned into an accelerator design for medium energy [22].

Published by the American Physical Society under the terms of the Creative Commons Attribution 4.0 International license. Further distribution of this work must maintain attribution to the author(s) and the published article's title, journal citation, and DOI.
Our approach starts from showing that a periodic grating provides phase dependent forces which can be concentrated in one kick per grating cell [23]. Per Panofsky-Wenzel theorem [24], this three-dimensional kick is irrotational, and can thus be modeled as a (time-dependent) potential in the Hamiltonian. Removing the time dependence is achieved by lattice integration of the linearized fields according to the Courant-Snyder (CS) theory [25]. An accelerator lattice design providing stable motion in the longitudinal and one transverse direction is obtained by an alternating phase focusing (APF) arrangement of grating segments treated as thick lenses [26]. Full scalability of the APF DLA is achieved by using a pulse-front-tilted laser [27-29] or an on-chip photonic waveguide system [30], which in principle allows us to work with arbitrary short pulses.

The equivalent magnetic focusing strength of an individual APF segment was predicted [26] and experimentally demonstrated to be in the MT/m range [31]. Moreover, the energy modulation of a subrelativistic DLA can also be turned into ballistic bunching [10,32-34]; however, the hereby created energy spread is too large to inject into a scalable APF DLA accelerator. A proposed APF-based segmented buncher [26] solves this problem and is currently being experimentally tested. Another ongoing experiment is the demonstration of a periodically segmented APF transport channel [35], which is however limited in length by the Rayleigh range of the electron beam in the invariant direction. In [26], we proposed to overcome this limit by installing an external quadrupole magnet which constantly focuses the beam in the vertical 
direction. A major challenge in the experimental realization of a fully scalable APF DLA is the alignment of this external magnet and the sufficient homogeneity of the laser fields in the invariant vertical direction of the structure.

In this Letter, we generalize the APF-based confinement to both transverse directions. This enables to completely eliminate external focusing devices in scalable DLAs of arbitrary length. Moreover, since the additional dimension provides an additional edge, the accelerating near-field is increased, which results in either higher gradient, or allows us to push for lower injection energy at given aperture. Previously reported minimal injection energies of $9.6 \mathrm{keV}$ [36] required using higher harmonics (in [36] the fifth) and confinement was not attained. Here, we intend to stay at the first harmonic since it provides the slowest dropoff from the grating surface and thus the highest center gradient. We show, that injection energies of 16.75 and $2.5 \mathrm{keV}$ are attainable at laser wavelengths of 2 and $6 \mu \mathrm{m}$, respectively. Thus, bulky high voltage feedthroughs in the experimental chambers can be entirely omitted.

The 3D APF-based DLA can still be fabricated by 2D lithographic techniques. The key idea is to work with two materials, exhibiting an as high as possible refractive index contrast, see Fig. 1. Such technology is already commercially available in nanoelectronics and -photonics, e.g., as silicon-on-insulator (SOI) wafers [37], and has been used to demonstrate a waveguide driven DLA recently [38]. The refractive indices at $2 \mu \mathrm{m}$ are [39] $n_{\mathrm{Si}}=3.67$ and $n_{\mathrm{SiO}_{2}}=1.44$, respectively. At first, we make the approximation of $n_{\mathrm{SiO}_{2}} \approx 1$, which will be later refined. In other words, the oxide serves as just a building brick to

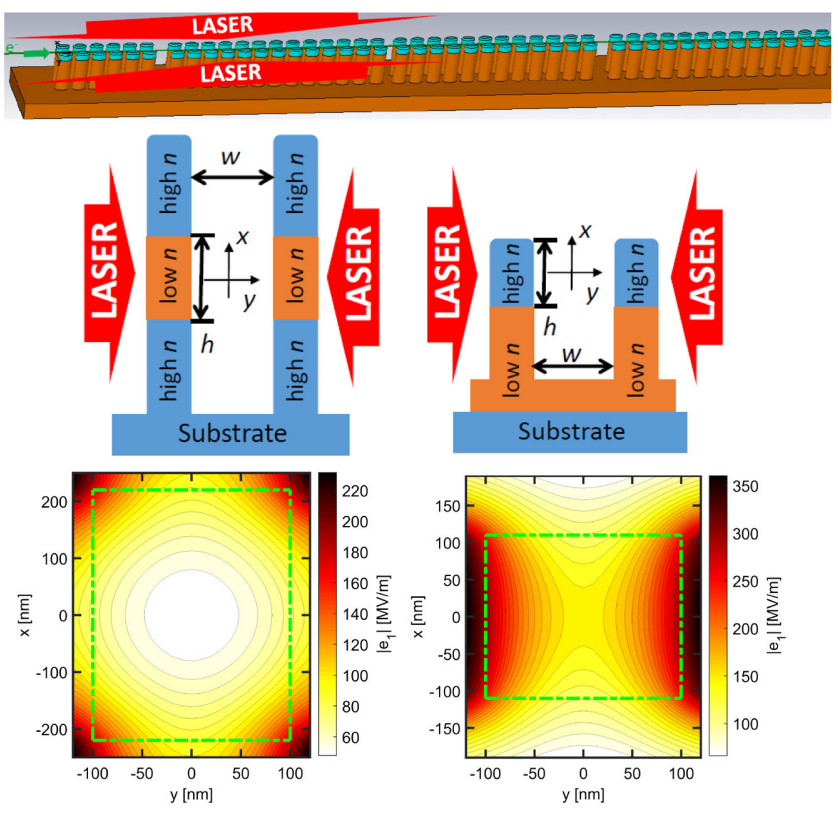

FIG. 1. Top: 3D APF DLA based on SOI dual pillars. Bottom: cross sections and $\left|e_{1}(x, y)\right|$ therein for in-phase APF (left) and counterphase APF (right, with approximation of $n_{\mathrm{SiO}_{2}} \approx 1$ ) and the beam channel $w \times h$ in green. construct 3D silicon structures by 2D lithography, where the layer thickness $h$ and the pillar semiaxis radii can attain single digit nanometer precisions.

To model the electromagnetic fields in the (quasi) periodic structures, we first look at the Helmholtz wave equation, in temporal frequency domain and Fourier series expanded in the longitudinal direction (see the Supplemental Material [40]),

$$
\left[\triangle_{\perp}-k_{z}^{2}+\omega^{2} / c^{2}\right] e_{1}(x, y)=0,
$$

where $\triangle_{\perp}$ is the transverse Laplacian, $k_{z}=\omega /(\beta c)$ is the longitudinal wave number of the synchronous mode $e_{1}$ to an electron traveling at speed $\beta c$ and $\omega=2 \pi c / \lambda$. Equation (1) is valid only in the vacuum of the beam channel. In contrast to conventional metallic accelerators, boundary conditions, necessary to solve Eq. (1), are not available. We can however determine the dispersion relation from Eq. (1) by $\partial_{x} \rightarrow-i k_{x}, \partial_{y} \rightarrow-i k_{y}$, and $\gamma^{2}=1 /\left(1-\beta^{2}\right)$ as

$$
k_{x}^{2}+k_{y}^{2}=\frac{\omega^{2}}{c^{2}}-k_{z}^{2}=-\frac{\omega^{2}}{\beta^{2} \gamma^{2} c^{2}}=:-\kappa^{2},
$$

which is plotted in Fig. 2. Instead of solving Eq. (1), we only need to determine $e_{10}=e_{1}(0,0)$ and one transverse wave number, which can be done numerically (see the Supplemental Material [40]), for each individual grating cell. The transverse dependence of $e_{1}$ can then be written analytically as

$$
e_{1}(x, y)=e_{10} \cosh \left(i k_{x} x\right) \cosh \left(i k_{y} y\right)
$$

which is numerically confirmed within $5 \%$ in the channel $w \times h$ and plotted in Fig. 1 over a slightly larger range. The assumption that the oxide can be neglected is crosschecked in Fig. 3 (top), where $\left|e_{10}\right|, k_{x}$, and $k_{y}$ are

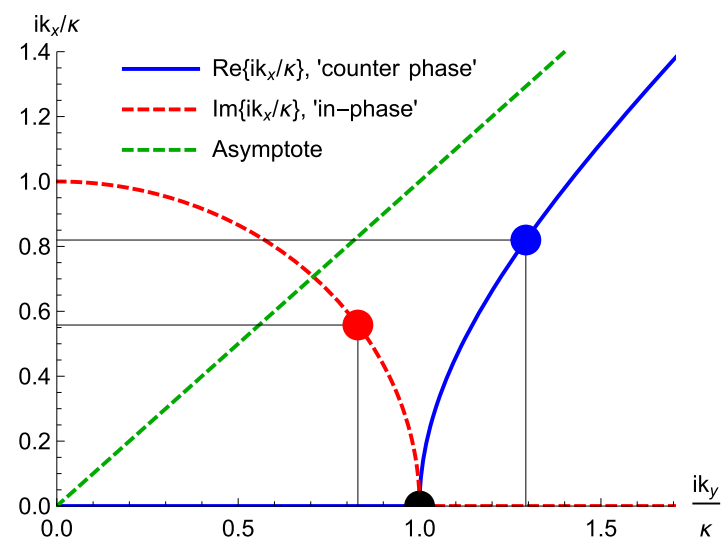

FIG. 2. Relation of $i k_{x}$ and $i k_{y}$. The black dot represents the two-dimensional APF scheme $\left(k_{x}=0\right)$ as introduced in [26]. The red and blue dots are examples of the in-phase and counterphase APF scheme, respectively. 

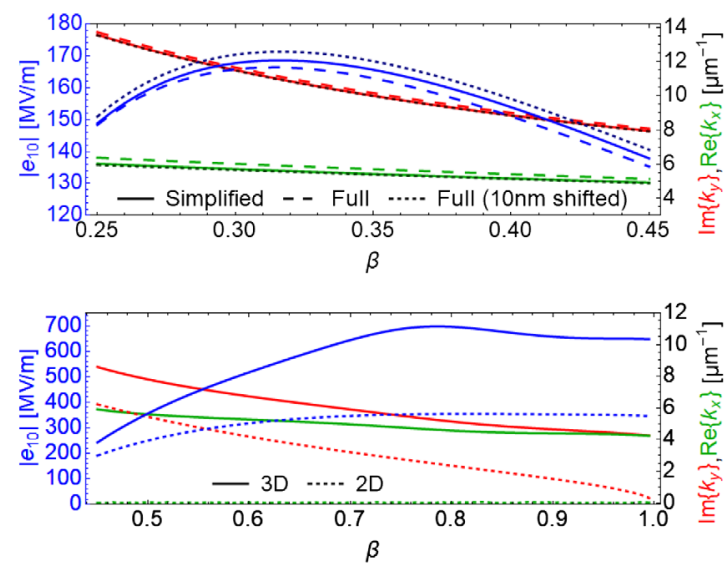

FIG. 3. Comparison of peak acceleration gradient and focusing strength of counterphase structures: (top panel) low energy for simplified vs full pillars and (bottom panel) high energy 3D vs $2 \mathrm{D}\left(k_{x}=0\right)$.

compared for a free-floating simplified pillar, a full pillar with the origin centered in $h$, and a full pillar with the origin slightly shifted towards the substrate. Since the curves agree to sufficient accuracy, we will continue with the simplified pillars for brevity. Figure 3 (bottom) shows a direct comparison of the peak gradient and focusing strength to the 2D case [26] for higher velocity, where an up to twofold gradient enhancement is visible. The enhancement is even larger at lower $\beta$, where the dropdown of $e_{1}$ renders the 2D scheme unfeasible.

For the laser traveling in $\pm y$ direction and polarized in $z$ direction, $i k_{y}$ is always a purely real number. However, $i k_{x}$ can either be purely real or purely imaginary, see Fig. 2. We will refer to these cases as in-phase and counterphase scheme, indicating whether or not both transverse planes are simultaneously focused.

With no loss of generality, we continue with the counterphase scheme only, since the structures are straightforward to fabricate on SOI wafers. The dependence of the wave numbers $k_{x / y}$ as well as $e_{10}$ on the height $h$ of a free-floating pillar is plotted in Fig. 4. The oscillation of $\left|e_{10}\right|$ is due to eigenmodes arising in the $x$ direction. Subsequently, the 2D case holds only for discrete values of $h$. These roots (and the corresponding maxima) of $k_{x}$ are not robust with respect to perturbations such as attaching the pillar to the oxide or to the substrate. Robust counterphase focusing behavior is found below the first root of $k_{x}$ and we choose $h=0.22 \mu \mathrm{m}$ (commercially available [37], vertical black line in Fig. 4), slightly below this first maximum.

In contrast to [26], the pillar shape is kept constant, i.e., $r_{z}=200 \mathrm{~nm}$ and $r_{y}=100 \mathrm{~nm}$ for Fig. 3 (top) and $r_{z}=400 \mathrm{~nm}$ and $r_{y}=300 \mathrm{~nm}$ for Fig. 3 (bottom), which set the minimum $\beta$ to roughly 0.25 and 0.45 , respectively. The occurring phase drift as function of $\beta=\lambda_{g} / \lambda$ is compensated by shifting the pillar center off the cell center by $\Delta z^{(n)}=\lambda_{g}^{(n)}\left[\arg \left(e_{10}^{(n)}\right)-\arg \left(e_{10}^{(1)}\right)\right] / 2 \pi$, see the

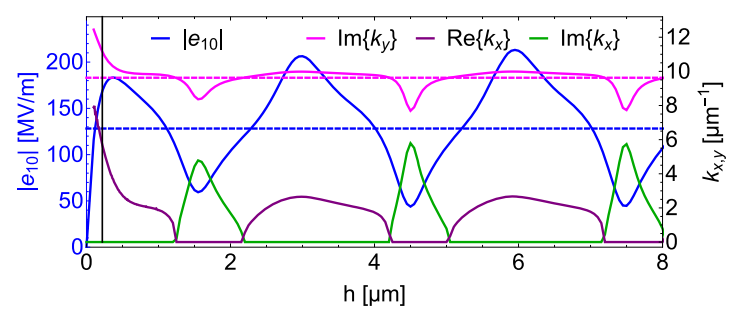

FIG. 4. Height scan for a free-floating dual pillar (counterphase) setup at $\beta=0.31$. Periodically reoccurring vertical eigenmodes make the $2 \mathrm{D}$ case (dashed lines) exceptional.

Supplemental Material [40]. The gradient can be further optimized by using a few additional pillar designs at different $\beta$. Moreover, fabrication errors for identical pillars are systematic and thus correctable, which decisively simplifies the fabrication and error estimation processes.

With the above knowledge of the electromagnetic field, we proceed to the Hamiltonian $H=\Delta \vec{P}^{2} /\left(2 m_{e} \gamma\right)+V$, where $\Delta \vec{P}=\left(p_{x}, p_{y}, \Delta p_{z} / \gamma\right)^{T}$ is the momentum deviation from the reference particle and $m_{e}$ is the electron rest mass. The time-dependent potential $V$ reads generally (same procedure as in [26]) as

$$
V(x, y, s)=q \operatorname{Im}\left\{k_{z}^{-1} e_{1}(x, y) e^{i k_{z} s}-i s e_{10} e^{i \varphi_{s}}\right\},
$$

where $q$ is the (negative) electron charge, $s$ is the relative longitudinal coordinate with respect to the laser phase, and $\varphi_{s}$ is the synchronous phase at which the reference particle gains energy according to the design acceleration ramp. Tracking with the nonlinear kicks according to Eq. (4) is performed by DLAtrack6D [23]. Expanding Eq. (4) to second order, Hamilton's equations provide Hill's equations

$$
\begin{gathered}
\Delta s^{\prime \prime}+K_{s} \Delta s=0, \\
y^{\prime \prime}+K_{y} y=0, \\
x^{\prime \prime}+K_{x} x=0,
\end{gathered}
$$

where $\Delta s=s-\lambda_{g} \varphi_{s} / 2 \pi$. Due to the absence of first order terms in $V$, the linearized motion is decoupled. The focusing functions are

$$
\begin{gathered}
K_{s}=-\frac{k_{z}^{2}}{\gamma^{2}} \frac{\left|q e_{10}\right|}{m_{e} \beta \gamma c \omega} \sin \left(\varphi_{s}\right), \\
K_{y}=\left(i k_{y}\right)^{2} \frac{\left|q e_{10}\right|}{m_{e} \beta \gamma c \omega} \sin \left(\varphi_{s}\right), \\
K_{x}=\left(i k_{x}\right)^{2} \frac{\left|q e_{10}\right|}{m_{e} \beta \gamma c \omega} \sin \left(\varphi_{s}\right),
\end{gathered}
$$




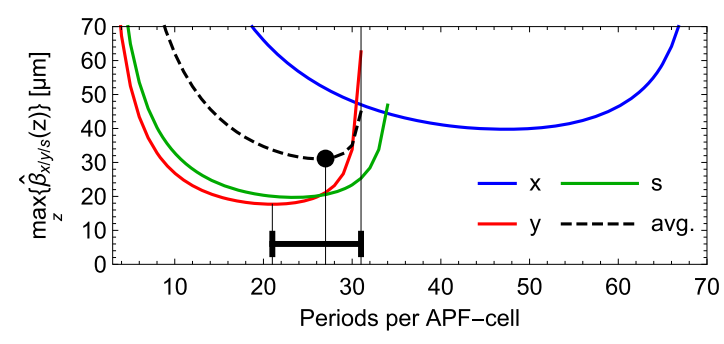

FIG. 5. Maximum of each $\hat{\beta}$ function for different APF cell lengths. The bar indicates the Pareto front of the multiminimization, where the minimum of the arithmetic average is taken as initial guess for every cell length in the design.

and fulfill $K_{x}+K_{y}+K_{s}=0$ according to Eq. (2), which reflects Earnshaw's theorem [44]. Note that $K_{s}$ is the same as $K$ in the 2D scheme [26] and the $s$ and $y$ planes are alternatingly focused by switching $\varphi_{s}$ using fractional period drifts. The numerical value of $K_{y}$ is however different from the 2D case and thus all three frequencies and phase advances are disparate. As compared to 2D, the 3D APF provides significantly higher values of $e_{10}, k_{x}$, and $k_{y}$, which allows us to push for lower injection energy while the confinement is maintained. Moreover, for $\beta \rightarrow 1, K_{x}$ and $K_{y}$ scale as $\gamma^{-1}$ since $k_{x}$ and $k_{y}$ remain constant, whereas in the 2D scheme a faster $\gamma^{-3}$ decay occurs (cf. Fig. 3).

This general description of the motion is now turned into a functioning accelerator that provides $3 \mathrm{D}$ particle confinement by individual CS lattice integration [25] in each plane $x, y, s$. As in [26], the lattice functions in Eqs. (6) are converted to CS-functions (also called Twiss parameters) $\eta=(\hat{\beta}, \hat{\alpha}, \hat{\gamma})^{T}$ by solving the Twiss map eigenvector problem $\eta_{0}=\mathbf{T} \eta_{0}$ for the initial values and subsequently mapping them to any other position.

The maxima of the $\hat{\beta}$ functions for a strictly periodic APF cell at $\beta=0.25$ are plotted in Fig. 5. To avoid electron loss at the aperture, the cell length of choice should minimize these three maxima at given laser amplitude. A suitable (not unique) multiobjective optimal cell length, i.e., an element of the Pareto front, is given by the minimum of the arithmetic average of these three maxima-curves.

At given injection energy we pick the synchronous phase as a compromise between desired acceleration gradient and required longitudinal focusing strength. The laser field strength is picked as slightly below the damage threshold fluence for a (curved-tilted) $100 \mathrm{fs}$ pulse. A laser amplitude of $500 \mathrm{MV} / \mathrm{m}$ from each side and a synchronous phase of $\pm 60^{\circ}$ off crest are chosen, leading to an average gradient of $G \approx \cos \left(\varphi_{s}\right)\left\langle\left|e_{10}\right|\right\rangle \approx 73 \mathrm{MeV} / \mathrm{m}$. Laser pulse shape and exact average gradient are detailed in the Supplemental Material [40].

The periodic solutions and their Twiss maps $\mathbf{T}_{P}^{x, y, s}$ for the optimal cell length as indicated in Fig. 5 are determined for a continuum of velocities $\beta$. Usually, a lattice obtained by simple matrix mapping of $\eta_{0}$ will exhibit growing $\hat{\beta}$ functions, due to cumulation of the small mismatch between two APF cells. Smooth and slowly growing $\hat{\beta}$ functions, such that the envelopes $a=(\hat{\beta} \varepsilon)^{1 / 2}$ are nongrowing, are obtained by manual correction of the segment lengths, which slightly squeezes the beam in one direction at the expense of another. Note that a slight growth of $\hat{\beta}$ is tolerable, since the emittance decreases by adiabatic
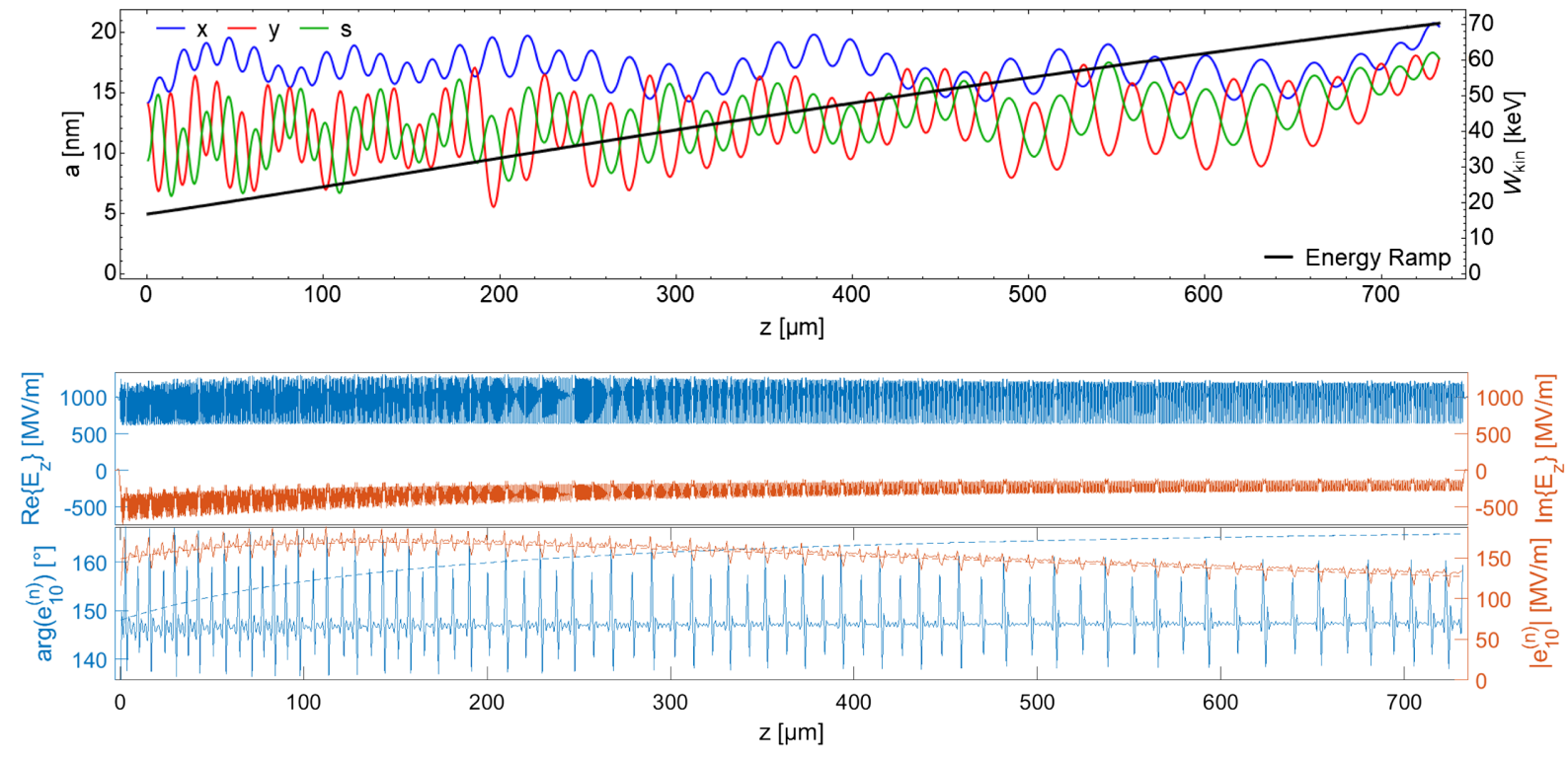

FIG. 6. Upper panel (design): envelopes for $\varepsilon_{n}=2.5 \mathrm{pm}$ and kinetic energy ramp. Lower panel (3D analysis): complex electric field $E_{z}(0,0, z)$ and spatial Fourier coefficients $e_{10}^{(n)}$ calculated from the full field windowed in each DLA cell $n$. The dashed lines represent the individually computed values of $e_{10}$ under periodic boundary conditions, which were used for the design. 


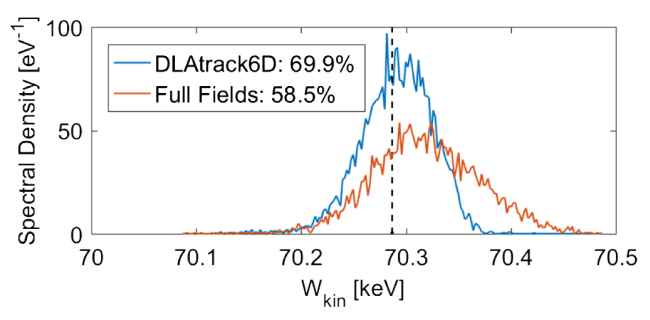

FIG. 7. Comparison of the final energy spectrum and throughput from a DLAtrack6D [23] simulation vs tracking simulation in the full laser fields using CST [45]. The dashed vertical line is the design top energy.

damping according to $\varepsilon=\varepsilon_{n} /(\beta \gamma)$, where the normalized emittance $\varepsilon_{n}$ is an invariant of the linearized motion.

The resulting energy ramp and envelopes for a structure that accelerates from $16.75 \mathrm{keV}(\beta=0.25)$ to $70.29 \mathrm{keV}$ $(\beta=0.48)$, consisting of 945 cells and 74 APF jumps, are plotted in Fig. 6 (top). After a full 3D field simulation [45], the complex field result along the channel is plotted below. Windowing this global field for each DLA cell allows a comparison between the $e_{10}^{(n)}$ in the entire accelerator to the individual $e_{10}^{(n)}$ initially computed under periodic boundary conditions (cf. Fig. 3). As visible, the phase drift compensation keeps $\arg \left(e_{10}^{(n)}\right)$ constant, but at the $\varphi_{s}$ jumps a Gibbs phenomenon occurs. This is the main source of excess emittance growth and particle loss (cf. Fig 7).

The injection parameters were chosen as Gaussian distributions with geometric emittances $\varepsilon_{x}=12 \mathrm{pm}$, $\varepsilon_{x}=7 \mathrm{pm}$, and bunch length $\sigma_{s}=5 \mathrm{~nm}$ with matched energy spread. These values are at the most sensitive beam loss clipping point and beam loss occurs in the lowest energy section of the accelerator. The throughput and energy spread is shown in Fig. 7 for one kick per cell vs 3D full field tracking. Similar results for $2.5 \mathrm{keV}$ $(\beta=0.1)$ to $16.75 \mathrm{keV}(\beta=0.25)$ using $\lambda=6 \mu \mathrm{m}$ are discussed in the Supplemental Material [40] and in principle, one could even start at a few eV only, by using a Terahertz driver. However, lowering the injection energy poses a challenge to the robustness. Structure bandwidth, fabrication tolerances, and injection energy mismatch have to be controlled more precise. Normalized emittances in the single digit picometer range are available [46,47], however, after the electrostatic preaccelerator mostly higher values are reported (e.g., [48,49]). This is due to nonlinear aberrations in the electrostatic lensing system. Our findings ease this problem significantly, since aberrations scale with the overall size of the system, which can be significantly reduced at lower injection energy. As confirmed by full 3D simulation, the 3D APF DLA scheme on SOI wafers is ready for experimental testing in different energy ranges. Moreover, DLAs with 6D confinement improve our ability to keep the energy spread small over long distance, e.g., to observe and control electron matter waves [50-54].
More detailed theoretical studies are required to assess the effects of nonlinear and coupled tune spread and emittance evolution, e.g., with the extended DLAtrack6D [55].

U.N. would like to thank Peyman Yousefi, Payton Broaddus, and Olav Solgaard for the discussions on SOI wafer processing. This work is funded by the Gordon and Betty Moore Foundation under Grant No. GBMF4744 (ACHIP).

*niedermayer@temf.tu-darmstadt.de

[1] A. Lohmann, Electron acceleration by light waves, IBM Tech. Note 5, 169 (1962).

[2] K. Shimoda, Proposal for an electron accelerator using an optical maser, Appl. Opt. 1, 33 (1962).

[3] J. Breuer and P. Hommelhoff, Dielectric laser acceleration of $28 \mathrm{keV}$ electrons with the inverse Smith-Purcell effect, Nucl. Instrum. Methods Phys. Res., Sect. A 740, 114 (2013).

[4] E. A. Peralta, K. Soong, R. J. England, E. R. Colby, Z. Wu, B. Montazeri, C. McGuinness, J. McNeur, K. J. Leedle, D. Walz, E. B. Sozer, B. Cowan, B. Schwartz, G. Travish, and R. L. Byer, Demonstration of electron acceleration in a laser-driven dielectric microstructure, Nature (London) 503, 91 (2013).

[5] K. P. Wootton, Z. Wu, B. M. Cowan, A. Hanuka, I. V. Makasyuk, E. A. Peralta, K. Soong, R. L. Byer, and R. J. England, Demonstration of acceleration of relativistic electrons at a dielectric microstructure using femtosecond laser pulses, Opt. Lett. 41, 2696 (2016).

[6] D. Cesar, S. Custodio, J. Maxson, P. Musumeci, X. Shen, E. Threlkeld, R. J. England, A. Hanuka, I. V. Makasyuk, E. A. Peralta, K. P. Wootton, and Z. Wu, High-field nonlinear optical response and phase control in a dielectric laser accelerator, Commun. Phys. 1, 46 (2018).

[7] P. Yousefi, J. McNeur, M. Kozák, U. Niedermayer, F. Gannott, O. Lohse, O. Boine-Frankenheim, and P. Hommelhoff, Silicon dual pillar structure with a distributed Bragg reflector for dielectric laser accelerators: Design and fabrication, Nucl. Instrum. Methods Phys. Res., Sect. A 909, 221 (2018).

[8] K. J. Leedle, R. Fabian Pease, R. L. Byer, and J. S. Harris, Laser acceleration and deflection of $963 \mathrm{keV}$ electrons with a silicon dielectric structure, Optica 2, 158 (2015).

[9] F. Krausz and M. Ivanov, Attosecond physics, Rev. Mod. Phys. 81, 163 (2009).

[10] Y. Morimoto and P. Baum, Diffraction and microscopy with attosecond electron pulse trains, Nat. Phys. 14, 252 (2018).

[11] A. H. Zewail and J. M. Thomas, 4D Electron Microscopy (Wiley-VCH Verlag GmbH \& Co. KGaA, Weinheim, 2010).

[12] R. K. Li and C.X. Tang, Temporal resolution of MeV ultrafast electron diffraction based on a photocathode RF gun, Nucl. Instrum. Methods Phys. Res., Sect. A 605, 243 (2009).

[13] S. P. Weathersby et al., Mega-electron-volt ultrafast electron diffraction at SLAC National Accelerator Laboratory, Rev. Sci. Instrum. 86, 073702 (2015). 
[14] N. Rubiano Da Silva, M. Möller, A. Feist, H. Ulrichs, C. Ropers, and S. Schäfer, Nanoscale Mapping of Ultrafast Magnetization Dynamics with Femtosecond Lorentz Microscopy, Phys. Rev. X 8, 031052 (2018).

[15] R. F. Egerton, Outrun radiation damage with electrons?, Adv. Struct. Chem. Imaging 1, 5 (2015).

[16] E. Slot, M. J. Wieland, G. de Boer, P. Kruit, G. F. ten Berge, A. M. C. Houkes, R. Jager, T. van de Peut, J. J. M. Peijster, S. W. H. K. Steenbrink, T. F. Teepen, A. H. V. van Veen, and B. J. Kampherbeek, MAPPER: High throughput maskless lithography, Emerging Lithogr. Technol. XII 6921, 69211P (2008).

[17] R. J. England et al., Dielectric laser accelerators, Rev. Mod. Phys. 86, 1337 (2014).

[18] R. B. Palmer, A laser driven grating linac, Part. Accel. 11, 375 (1980), http://cds.cern.ch/record/1107986/files/p81.pdf.

[19] K. Kim and N. Kroll, Some effects of the transverse-stability reqirement on the design of a grating linac, Report No. LBL14378, Cornell University, Ithaca, NY, 1982.

[20] M. Pickup, A grating linac at microwave frequencies, Report No. CLNS-85/655, Lawrence Berkeley Laboratory, UC Berkeley California, 1985.

[21] B. Naranjo, A. Valloni, S. Putterman, and J. B. Rosenzweig, Stable Charged-Particle Acceleration and Focusing in a Laser Accelerator using Spatial Harmonics, Phys. Rev. Lett. 109, 164803 (2012).

[22] D. Cesar, P. Musumeci, and J. England, All optical control of beam dynamics in a DLA, in 2018 IEEE Advanced Accelerator Concepts Workshop (AAC), Breckenridge, CO, USA (IEEE, Breckenridge, 2018), pp. 1-5.

[23] U. Niedermayer, T. Egenolf, and O. Boine-Frankenheim, Beam dynamics analysis of dielectric laser acceleration using a fast $6 \mathrm{D}$ tracking scheme, Phys. Rev. Accel. Beams 20, 111302 (2017).

[24] W. K. H. Panofsky and W. A. Wenzel, Some considerations concerning the transverse deflection of charged particles in radio-frequency fields, Rev. Sci. Instrum. 27, 967 (1956).

[25] E. Courant and H. Snyder, Theory of the alternatinggradient synchrotron, Ann. Phys. (N.Y.) 3, 1 (1958).

[26] U. Niedermayer, T. Egenolf, O. Boine-Frankenheim, and P. Hommelhoff, Alternating-Phase Focusing for DielectricLaser Acceleration, Phys. Rev. Lett. 121, 214801 (2018).

[27] Y. Wei, M. Ibison, G. Xia, J. D. A. Smith, and C. P. Welsch, Dual-grating dielectric accelerators driven by a pulse-fronttilted laser, Appl. Opt. 56, 8201 (2017).

[28] D. Cesar, J. Maxson, P. Musumeci, X. Shen, R. J. England, and K. P. Wootton, Optical design for increased interaction length in a high gradient dielectric laser accelerator, Nucl. Instrum. Methods Phys. Res., Sect. A 909, 252 (2018).

[29] M. Kozák, J. McNeur, N. Schönenberger, J. Illmer, A. Li, A. Tafel, P. Yousefi, T. Eckstein, and P. Hommelhoff, Ultrafast scanning electron microscope applied for studying the interaction between free electrons and optical near-fields of periodic nanostructures, J. Appl. Phys. 124, 023104 (2018).

[30] T. W. Hughes, S. Tan, Z. Zhao, N. V. Sapra, K. J. Leedle, H. Deng, Y. Miao, D. S. Black, O. Solgaard, J. S. Harris, J. Vuckovic, R. L. Byer, S. Fan, R. J. England, Y. J. Lee, and M. Qi, On-Chip Laser-Power Delivery System for Dielectric Laser Accelerators, Phys. Rev. Applied 9, 054017 (2018).
[31] D. S. Black, K. J. Leedle, Y. Miao, U. Niedermayer, R. L. Byer, and O. Solgaard, Laser-Driven Electron Lensing in Silicon Microstructures, Phys. Rev. Lett. 122, 104801 (2019).

[32] U. Niedermayer, O. Boine-Frankenheim, and T. Egenolf, Designing a dielectric laser accelerator on a chip, J. Phys. Conf. Ser. 874, 012041 (2017).

[33] D. S. Black, U. Niedermayer, Y. Miao, Z. Zhao, O. Solgaard, R. L. Byer, and K. J. Leedle, Net Acceleration and Direct Measurement of Attosecond Electron Pulses in a Silicon Dielectric Laser Accelerator, Phys. Rev. Lett. 123, 264802 (2019).

[34] N. Schönenberger, A. Mittelbach, P. Yousefi, J. McNeur, U. Niedermayer, and P. Hommelhoff, Generation and Characterization of Attosecond Microbunched Electron Pulse Trains via Dielectric Laser Acceleration, Phys. Rev. Lett. 123, 264803 (2019).

[35] U. Niedermayer et al., Challenges in simulating beam dynamics of dielectric laser acceleration, Int. J. Mod. Phys. A 34, 1942031 (2019).

[36] J. McNeur, M. Kozak, D. Ehberger, N. Schönenberger, A. Tafel, A. Li, and P. Hommelhoff, A miniaturized electron source based on dielectric laser accelerator operation at higher spatial harmonics and a nanotip photoemitter, J. Phys. B 49, 034006 (2016).

[37] www.order.universitywafer.com, 2019.

[38] N. V. Sapra, K. Y. Yang, D. Vercruysse, K. J. Leedle, D. S. Black, R. J. England, L. Su, R. Trivedi, Y. Miao, O. Solgaard, R. L. Byer, and J. Vuckovic, On-chip integrated laser-driven particle accelerator, Science 367, 79 (2020).

[39] www.refractiveindex.info, 2019.

[40] See the Supplemental Material at http://link.aps.org/ supplemental/10.1103/PhysRevLett.125.164801 for details on structure constant, laser pulse shaping, an ultralow injection energy design, and video examples, which contains Refs. [41-43].

[41] P. Pronko, P. V. Rompay, C. Horvath, X. Liu, T. Juhasz, and G. Mourou, Avalanche ionization and dielectric breakdown in silicon with ultrafast laser pulses, Phys. Rev. B 58, 2387 (1998).

[42] K. Soong, R. L. Byer, E. R. Colby, R. J. England, and E. A. Peralta, Laser damage threshold measurements of optical materials for direct laser accelerators, AIP Conf. Proc. 1507, 511 (2012).

[43] B. Sun, P. S. Salter, and M. J. Booth, Pulse front adaptive optics: a new method for control of ultrashort laser pulses, Opt. Express 23, 19348 (2015).

[44] S. Earnshaw, On the nature of the molecular forces which regulate the constitution of the luminferous ether, Trans. Cambridge Philos. Soc. 7, 97 (1842).

[45] CST, CST Studio Suite (2019).

[46] D. Ehberger, J. Hammer, M. Eisele, M. Krüger, J. Noe, A. Högele, and P. Hommelhoff, Highly Coherent Electron Beam from a Laser-Triggered Tungsten Needle Tip, Phys. Rev. Lett. 114, 227601 (2015).

[47] A. Feist, N. Bach, N. Rubiano da Silva, T. Danz, M. Möller, K. E. Priebe, T. Domröse, J. G. Gatzmann, S. Rost, J. Schauss, S. Strauch, R. Bormann, M. Sivis, S. Schäfer, and C. Ropers, Ultrafast transmission electron microscopy using a laser-driven 
field emitter: Femtosecond resolution with a high coherence electron beam, Ultramicroscopy 176, 63 (2017).

[48] A. Tafel, S. Meier, J. Ristein, and P. Hommelhoff, Femtosecond Laser-Induced Electron Emission from Nanodiamond-Coated Tungsten Needle Tips, Phys. Rev. Lett. 123, 146802 (2019).

[49] T. Hirano, K. E. Urbanek, A. C. Ceballos, D. S. Black, Y. Miao, R. Joel England, R. L. Byer, and K. J. Leedle, A compact electron source for the dielectric laser accelerator, Appl. Phys. Lett. 116, 161106 (2020).

[50] B. Barwick, D. J. Flannigan, and A. H. Zewail, Photoninduced near-field electron microscopy, Nature (London) 462, 902 (2009).

[51] O. Keller, Quantum Theory of Near Fields (Springer, Berlin-Heidelberg, 2011).
[52] E. Jones, M. Becker, J. Luiten, and H. Batelaan, Laser control of electron matter waves, Laser Photonics Rev. 10, 214 (2016).

[53] G. M. Vanacore, I. Madan, G. Berruto, K. Wang, E. Pomarico, R. J. Lamb, D. McGrouther, I. Kaminer, B. Barwick, F. J. García De Abajo, and F. Carbone, Attosecond coherent control of free-electron wave functions using semi-infinite light fields, Nat. Commun. 9, 2694 (2018).

[54] S. Nehemia, R. Dahan, M. Shentcis, O. Reinhardt, Y. Adiv, K. Wang, O. Beer, Y. Kurman, X. Shi, M. H. Lynch, and I. Kaminer, Observation of the stimulated quantum Cherenkov effect, arXiv:1909.00757.

[55] T. Egenolf, U. Niedermayer, and O. Boine-Frankenheim, Tracking with wakefields in dielectric laser acceleration grating structures, Phys. Rev. Accel. Beams 23, 054402 (2020). 